\title{
Value of Maximum Standard Uptake Value as a Positron Emission Tomography/Computed Tomography Index in the Diagnosis and Prognosis of Patients with Gastric Cancer
}

\section{Kairui Wang}

School of Clinical Medicine, Ningxia Medical University

\section{Baozhen Wang}

School of Clinical Medicine, Ningxia Medical University

\section{Qian Zhao}

Department of Nuclear Medicine, General Hospital of Ningxia Medical University

\section{Li Guo}

School of Clinical Medicine, Ningxia Medical University

\section{Yongfeng Liang}

School of Clinical Medicine, Ningxia Medical University

\section{Duowen Zhao}

Gansu Provincial Maternity and Child-care Hospital

\section{Jing Chen}

Department of Medical Genetic and Cell Biology, Ningxia Medical University

Tao Li ( $D$ lit1979@163.com )

https://orcid.org/0000-0003-1634-0889

Research article

Keywords: gastric cancer, primary gastric lymphoma, PET/CT, diagnosis, prognosis

Posted Date: April 1st, 2021

DOl: https://doi.org/10.21203/rs.3.rs-378413/v1

License: (c) (i) This work is licensed under a Creative Commons Attribution 4.0 International License. Read Full License 


\section{Abstract}

Background: To study the application value of standard uptake value (SUVmax), a positron emission tomography/computed tomography (PET/CT) index in the diagnosis and evaluation of the prognosis of patients with gastric cancer who have not received any treatment.

Methods: A retrospective analysis was made on the patients who were diagnosed to have gastric cancer at the General Hospital of Ningxia Medical University and received a PET/CT examination prior to treatment. According to different factors like sex, pathological stage, and survival time, the SUVmax in the results of ${ }^{18}$ F-labelled fluoro-2-deoxyglucose (18F-FDG) PET/CT examination was statistically compared and analysed.

Results: A total of 110 newly diagnosed patients with gastric cancer were included in this study. Pathological results confirmed that there were 78 cases of gastric adenocarcinoma, 30 cases of primary gastric lymphoma and two cases of chronic atrophic gastritis. The difference between the primary gastric lymphoma group and gastric adenocarcinoma groups was statistically significant $(t=4.13, P<0.05)$. In the postoperative pathological report of patients with gastric cancer, the SUVmax of stage I, stage II, stage III and stage IV were $2.89 \pm 1.36,14.09 \pm 9.32,7.36 \pm 3.72$, and $10.20 \pm 1.91$, respectively, the difference between each group was statistically significant $(F=16.10, P<0.05)$. The univariate survival analysis showed that there was a significant difference in survival time between the low SUVmax and high SUVmax groups $\left(X^{2}=5.08, P<0.05\right)$. Cox multivariate survival analysis showed that SUVmax was an independent factor affecting postoperative survival time of patients with gastric adenocarcinoma $(\mathrm{P}<$ 0.05). Compared with the low SUVmax group, patients in the high SUVmax group had a higher postoperative death risk than those in the low SUVmax group, hazard ration (HR) $=3.91$ (95\% confidence interval [Cl]: 1.09-14.01).

Conclusion: The index of SUVmax in PET/CT can provide a reliable semi-quantitative diagnostic value for pathological staging after gastric cancer surgery. Furthermore, SUVmax has an important reference value for the survival time of patients after gastric cancer surgery, which can better guide clinical treatment.

\section{Introduction}

For a long time, attention has been given to gastric cancer as the most common digestive tract tumour, and a great deal of energy and financial resources have been devoted to explore the best measures in its diagnosis, treatment, and prevention. China is one of the countries with the highest incidence of gastric cancer, which is different from other countries in epidemiology, clinical pathology, and treatment modality [1]. Seventy percent of new cases of gastric cancer occurred in developing countries, and China accounted for $42.6 \%$ [2]. In China, the data of the National Cancer Centre in 2018 show that [3], although the overall incidence of gastric cancer is decreasing every year, its mortality rate is still high, and has the second highest mortality rate among malignant tumours in China, only second to lung cancer. Gastric cancer is also one of the most common digestive tract tumours worldwide. In recent years, with the 
development of multidisciplinary comprehensive treatment in various countries around the world, the survival rate of patients with gastric cancer has been greatly improved. However, in the Asian population, it is still one of the most common malignant tumours leading to cancer-related deaths [4-5]. According to reports, advanced gastric cancer has become the second leading cause of cancer-related death worldwide [6-8]. Therefore, early detection and early diagnosis are important factors for the diagnosis and treatment of gastric cancer. The traditional diagnosis of gastric cancer still takes pathological biopsy under a gastroscope as the gold standard, and gastric computed tomography (CT) and other examinations as auxiliary examination methods. At present, due to the update of medical methods, fluoro-deoxyglucose positron emission CT (18F-FDG PET/CT) has gradually been recognized and become an important examination method chosen by most patients with tumours and doctors. Studies have shown that it can prompt the glucose metabolism of lesions from the aspect of functional imaging, supplemented by anatomical observation [9]. Now, PET/CT has become an indispensable examination method for the diagnosis, staging and curative effect evaluation of many types of malignant tumours. Almuhaimid et al. [10] analysed 75 patients with highly differentiated salivary adenocarcinoma through an observational study, and found that when SUVmax $>4.3$ and mean standardized uptake value (SUV mean) $>3.0$, there was a significant correlation with progression free survival (PFS), thus confirming the prognostic value of PET/CT for patients with highly differentiated salivary adenocarcinoma. Gao Zhongshan [11] et al. found that the diagnostic accuracy, sensitivity, and specificity of the observation group were $93.33 \%, 75.68 \%$, and $45.45 \%$, which were higher than those of the control group when SUVmax $>2.5$ was used as the auxiliary condition for diagnosing prostate cancer. Therefore, SUVmax can be used as a diagnostic method for prostate cancer. Currently, with further exploration of immunotherapy for gastric cancer and tumour microenvironment, various biological substances that suggest the existence of tumours are becoming more and more abundant, which is a favourable discovery for gastric cancer and other malignant tumours. Most importantly, the combination of this targeted research and $\mathrm{PET} / \mathrm{CT}$ also makes the latter play an increasingly important role. In gastric cancer, PET/CT plays an important role in metastasis and recurrence after radical operation and evaluation of the curative effect after radiotherapy and chemotherapy, but the application value of PET/CT before surgery has been in dispute [12]. Therefore, this study aimed to explore the clinical diagnostic and prognostic value of $\mathrm{PET} / \mathrm{CT}$ in patients with gastric cancer prior to any treatment.

\section{Materials And Methods}

\section{Patients}

A retrospective analysis was carried out on the patients who were newly diagnosed with gastric cancer and underwent PET/CT examination between May 8, 2012 and July 14, 2020 in the Ningxia Medical University General Hospital. Criteria for inclusion of the patients for this clinical study were as follows: (1) patients who had a clear pathological results report; (2) patients who had not received chemotherapy, radiotherapy, and radiofrequency ablation before admission; (3) patients who had no previous history of other malignant tumours; and (4) patients with complete data. Exclusion criteria in this study were as 
follows: (1) tumour remaining at the gastroesophageal junction; (2) metastases to the stomach from primary malignant tumours in other parts; and (3) patients with heart, liver, kidney and other important organ dysfunction. After the above inclusion and exclusion criteria, 110 eligible cases were included in this study for analyses. All 110 patients were examined by abdominal CT (or abdominal enhanced CT) and PET/CT within 1-2 weeks prior to treatment. There were 72 males and 38 females, with age ranging from 20 to 89 years, with an average age of 61 years and a median age of 63 years. Pathological reports confirmed that there were 78 cases of gastric adenocarcinoma, 30 cases of primary gastric lymphoma and two cases of chronic atrophic gastritis. The postoperative pathological stages of patients with gastric cancer were divided according to the guidelines for diagnosis and treatment of gastric cancer of Chinese Society of Clinical Oncology [13].

\section{8f-fdg Pet/ct Imaging}

The Discovery VCT 64 PET/CT scanner produced by GE company of America was used. All patients were fasted for $6 \mathrm{~h}$ or more, and their blood sugar was less than $7 \mathrm{mmol} / \mathrm{L}$. 18F-FDG $5 \mathrm{MBq} / \mathrm{kg}$ was injected intravenously in a quiet and dark environment, and a whole-body PET imaging was performed after lying down for $40 \mathrm{~min}$. First, CT was used for helical scanning, covering all tissues from the top of the skull to the thighs. The acquisition conditions were as follows: CT tube voltage: $120 \mathrm{kV}$; CT tube current: 25$210 \mathrm{~mA}$ (automatic); rotation time of one circle of the bulb: $0.5 \mathrm{~s}$; Spiral scanning thickness: $3.75 \mathrm{~mm}$; screw pitch: $0.516: 1 \mathrm{~mm}$. Then, a three-dimensional acquisition method was adopted, and the normal acquisition time of each group of images was 2 min per bed. The reconstruction method of PET is a traditional iterative method, with 28 subsets and two iterations. Finally, after correction and fusion, crosssectional, coronal, and sagittal images were obtained. The image data of all patients were double-blindly reviewed by two experienced nuclear medicine physicians. First, the uptake degree of the lesion tissue was visually analysed, and it was regarded as positive if it was higher than the surrounding normal tissue. Then, the region of interest (ROI) was delineated at the lesion site and SUVmax was measured. Finally, the benign and malignant lesions were judged according to SUVmax and thickening degree of the lesion site. Gastric CT (or abdominal enhanced CT) review standard takes into account positive indicators such as gastric wall thickening $>3 \mathrm{~mm}$, unclear tissue level and local enhanced imaging, and finally judges the benign and malignant lesions. There was little difference in the results of the two reviewers in this study. When there was difference in the reading results of the two physicians, consistent results were obtained after repeated reading, participating in a discussion and combining with the above review criteria.

\section{Follow-up}

Through evaluation and screening, 110 patients with gastric cancer were included in this study. Among them, 36 patients received surgical treatment, which consisted only of radical gastrectomy. The 36 patients were followed up by telephone. The follow-up started on the first day after surgery and ended on 
July 27, 2020. Survival time was defined as the time from the start of follow-up to the occurrence of death or the deadline of follow-up.

\section{Statistical analysis}

Statistical software SPSS v.22.0 was used for data analyses. The data conforming to normal distribution were compared by independent sample t-test and one-way ANOVA and expressed as mean \pm standard deviation. Non-parametric tests were used to compare the differences among groups for data that did not conform to normal distribution. X-tile statistical software was used to determine the best cut-off point of SUVmax [14], and the Kaplan-Meier and Cox survival analyses were used to analyse the factors affecting postoperative survival time, and differences with a $p<0.05$ were considered statistically significant.

\section{Results}

Comparison of gastric CT (or abdominal enhanced CT) and PET/CT for benign and malignant gastric lesions

A total of 110 patients were examined by gastric CT (or abdominal enhanced CT) and PET/CT, respectively. According to the results of pathological reports, 108 cases were malignant gastric tumours (including gastric adenocarcinoma and primary gastric lymphoma), and two cases were benign gastric lesions, all of which were chronic atrophic gastritis. The sensitivity, specificity, and accuracy of gastric CT (or abdominal enhanced CT) and PET/CT are shown in Table 1.

Table 1

Comparison of the results of gastric CT (or abdominal enhanced CT) and PET/CT

\begin{tabular}{|c|c|c|c|}
\hline & sensitivity & Specificity & Accuracy \\
\hline ECT & $88.0 \%(95 / 108)$ & $100 \%(2 / 2)$ & $88.2 \%(97 / 110)$ \\
\hline PET/CT & $98.1 \%(106 / 108)$ & $100 \%(2 / 2)$ & $98.2 \%(108 / 110)$ \\
\hline
\end{tabular}

The relationship between SUVmax and primary gastric lymphoma and gastric adenocarcinoma

There were 78 cases of gastric adenocarcinoma and 30 cases of primary gastric lymphoma in patients with gastric malignant tumours confirmed by pathological results. the SUVmax results of PET/CT examination were extracted and sorted out, and the data between the two groups were statistically analysed. The results are shown in Table 2. It can be seen that the SUVmax of the primary gastric lymphoma group is $15.87 \pm 10.22$, and that of gastric adenocarcinoma group is $7.74 \pm 5.51$, with a statistically significant difference $(t=4.13, p<0.05)$. 
Table 2

Relationship between SUVmax and primary gastric lymphoma and gastric adenocarcinoma in 108 cases

\begin{tabular}{|lllll|}
\hline Pathological types & Number of cases & Mean \pm standard deviation & $\mathbf{t}$ & $\boldsymbol{P}$ \\
\hline Gastric adenocarcinoma & 78 & $7.74 \pm 5.51$ & 4.13 & 0.00 \\
\hline Primary gastric lymphoma & 30 & $15.87 \pm 10.22$ & & \\
\hline SUVmax, maximum standard uptake value & & & \\
\hline
\end{tabular}

The relationship between the SUVmax value and clinicopathological features of gastric adenocarcinoma

A further analysis of the medical records of 110 patients who were diagnosed with gastric cancer for the first time and examined before treatment showed that 36 patients were treated by radical gastrectomy in our hospital. These patients who received surgical treatment were confirmed to have gastric adenocarcinoma according to the results of postoperative pathological reports. Among them, there were 28 male patients and eight female patients. The patients ranged in age from 43 to 84 years, with an average age of 64 years and a median age of 64 years. The relationship between the clinicopathological features and SUVmax is shown in Table 3, and the differences between SUVmax and age, tumour location, pathological stage and T stage are statistically significant $(p<0.05)$. 
Table 3

Relationship between clinicopathological features and SUVmax in 36 cases of gastric adenocarcinoma

\section{Variables}

Age

$\llbracket 65$

$\geq 65$

Sex

Male

Female

Tumour site

Gastric fundus

Gastric body

Gastric antrum

Histological type

High differentiation

Moderate differentiation

Low differentiation

Pathological stage

I

II

III

IV

T stage

T1

T2

T3

T4

Lymph node metastasis n Mean \pm standard deviation

$\mathrm{t} / \mathrm{F}$

2.73

$19 \quad 5.86 \pm 3.61$

$17 \quad 11.65 \pm 8.04$

0.10

0.92

$8 \quad 8.81 \pm 8.92$

$6.24 \quad 0.00$

$13 \quad 13.19 \pm 8.58$

$15 \quad 5.79 \pm 2.95$

$8 \quad 6.40 \pm 4.37$

1.67

0.20

$5 \quad 5.26 \pm 3.19$

$13 \quad 11.02 \pm 6.63$

$18 \quad 7.77 \pm 7.10$

$16.10 \quad 0.00$

$7 \quad 2.89 \pm 1.36$

$10 \quad 14.09 \pm 9.32$

$16 \quad 7.36 \pm 3.72$

$3 \quad 10.20 \pm 1.91$

7.69

0.00

$4 \quad 2.83 \pm 1.62$

$7 \quad 6.63 \pm 6.11$

$8 \quad 13.85 \pm 10.11$

$17 \quad 8.29 \pm 3.87$

1.67

0.10

Note: The statistical value of age group, sex group and lymph node metastasis group are $t$ values, and that of the other groups are $F$ values. 


\begin{tabular}{|lcll|}
\hline Variables & $\mathbf{n}$ & Mean \pm standard deviation & t/F \\
\hline Positive & 25 & $9.80 \pm 7.17$ \\
Negative & 11 & $5.86 \pm 4.68$ \\
\hline $\begin{array}{l}\text { Note: The statistical value of age group, sex group and lymph node metastasis group are t values, } \\
\text { and that of the other groups are F values. }\end{array}$ \\
\hline
\end{tabular}

SUVmax, maximum standard uptake value

\section{The relationship between SUVmax value and survival time in gastric adenocarcinoma}

In this study, 36 patients with gastric adenocarcinoma who received radical gastrectomy were followedup by telephone. One patient was lost follow-up due to incorrect contact information, two patients were lost follow-up due to telephone failure, and the remaining 33 patients successfully completed follow-up work, with a median follow-up time of 33 months. The SUVmax data of the 33 patients with PET/CT were extracted and sorted, and the best limit point of SUVmax was determined to be 9.0 by the X-tile statistical software. Therefore, SUVmax $<9.0$ was considered as the low SUVmax group, and SUVmax $\geq 9.0$ as the high SUVmax group. The results of the Kaplan-Meier univariate prognosis analysis of 36 patients with gastric adenocarcinoma after surgery are shown in Table 4 and Fig. 1. The results of Cox multivariate survival analysis are shown in Table 5 and Fig. 2. 
Table 4

Univariate analysis of survival time of 33 patients with gastric adenocarcinoma after surgery

\begin{tabular}{|c|c|c|c|c|}
\hline Variables & $\mathbf{n}$ & Survival time, (months) & $\mathrm{X} 2$ & $P$ \\
\hline Age & & & 1.94 & 0.16 \\
\hline$₫ 65$ & 17 & 20.76 & & \\
\hline$\geq 65$ & 16 & 27.13 & & \\
\hline Sex & & & 1.34 & 0.25 \\
\hline Male & 27 & 22.37 & & \\
\hline Female & 6 & 30.50 & & \\
\hline Differentiation degree & & & 0.37 & 0.54 \\
\hline Poor differentiation & 17 & 25.35 & & \\
\hline Good differentiation & 16 & 22.25 & & \\
\hline SUVmax & & & 5.08 & 0.02 \\
\hline Low SUVmax & 22 & 27.77 & & \\
\hline High SUVmax & 11 & 16.00 & & \\
\hline Tumour site & & & 1.04 & 0.60 \\
\hline Gastric fundus & 12 & 28.33 & & \\
\hline Gastric body & 14 & 20.71 & & \\
\hline Gastric antrum & 7 & 22.43 & & \\
\hline Lymph node metastasis & & & 2.16 & 0.14 \\
\hline Positive & 23 & 21.35 & & \\
\hline Negative & 10 & 29.60 & & \\
\hline
\end{tabular}

Table 5

Multivariate analysis of the survival time of 33 patients with gastric adenocarcinoma after surgery

\begin{tabular}{|llllll|}
\hline Variables & B & SE & Wald & HR & $\boldsymbol{P}$ \\
\hline SUVmax & 1.36 & 0.65 & 4.36 & $3.91(1.09-14.01)$ & 0.04 \\
\hline
\end{tabular}


Gastric malignant tumour, its morbidity and mortality have been ranked high all the time in the world. According to statistics, as the most common malignant tumour of the digestive system, the five-year survival rate of gastric cancer is between $20 \%$ and $30 \%$ [15]. In China, the mortality rate of gastric cancer ranks second [16]. With the change of the living environment and diet structure of individuals, the incidence rate of gastric cancer is increasing every year. Therefore, early diagnosis and accurate staging of gastric cancer are particularly important, which can directly affect the treatment and prognosis of patients [17]. Accurate staging (including the scope of local invasion, lymph node metastasis and distant metastasis) can optimize the treatment plan and guide surgery [18]. Due to its high spatial and density resolution, enhanced CT has become a positive and extensive preoperative staging method for gastric cancer [19]. Admittedly, gastroscopy is one of the most effective methods for early gastric cancer examination, and its status as the gold standard cannot be shaken. However, the gastroscope can only observe the local lesions on the surface of gastric mucosa, which still lacks the analysis of the depth of tumour invasion, lymph node metastasis and invasion and metastasis of adjacent organs. Moreover, the gastroscope, as an invasive examination, causes pain to patients, and its results will also be affected more or less by the accuracy of sampling materials. At present, the treatment methods of cancer tend to be individualized and precise medical treatment and multidisciplinary comprehensive treatment [20].

With the continuous improvement of medical level and updating of medical equipment, PET/CT has been widely used to evaluate the metastasis and staging of malignant tumours [21-22]. In gastric cancer, the degree of glucose transporter 1 (GLUT1) expression is also different in different pathological types. The expression level of GLUT1 in highly differentiated gastric cancer cells is higher than that in gastric signetring cell carcinoma, therefore, the latter has lower results for 18F-FDG uptake [23]. In addition, compared with other histological types of gastric cancer, pyruvate kinase M2 (PKM2) subtype in signet-ring cell carcinoma is at a low expression level [24]. All these factors lead to the poor prognosis of signet-ring cell carcinoma, but the uptake of 18F-FDG is less, which makes it express low SUV results. Therefore, many clinicians and researchers are sceptical about the diagnostic value of PET/CT in gastric cancer. This research is based on the results of a retrospective study to illustrate this issue.

The results of this retrospective study showed that the sensitivity and accuracy of gastric CT (or abdominal enhanced CT) in distinguishing benign and malignant gastric lesions are lower than that of $\mathrm{PET} / \mathrm{CT}$. In this study, there were two false-negative results on PET/CT. Pathological results confirmed that the proportion of mucinous adenocarcinoma was high or it was signet ring cell carcinoma, which was considered to be related to the low expression of GLUT-1 and PKM2, so that the intake was low and the SUVmax was small, therefore, a false negative result appeared. Considering that the proportion of gastric signet-ring cell carcinoma was relatively small after all, the diagnostic advantages of PET/CT in this aspect will be more obvious when the sample size is large.

A total of 108 patients were confirmed to have gastric malignant tumours by pathological results, including 78 cases of gastric adenocarcinoma and 30 cases of primary gastric lymphoma. The difference between the primary gastric lymphoma and gastric adenocarcinoma groups was statistically significant $(t=4.13, P<0.05)$. Sun Gaofeng et al. [25] studied the PET/CT results of 43 patients with 
gastric cancer and 13 patients with primary gastric lymphoma and found that there was a difference in SUVmax between them, and the P $<0.05$. Similarly, Li XF [26] et al. retrospectively analysed 73 cases of gastric cancer and 52 cases of gastric lymphoma, and found that compared with gastric cancer, the results of PET/CT examination of gastric lymphoma were different, and higher SUVmax suggested that gastric lymphoma was more likely. The above results are consistent with the results of the present study. Clinically, for patients with possible lymphoma, when evaluating extra-nodal lymph nodes prior to treatment, apart from the invasive operation of tissue biopsy, the most common inspection method is computer imaging, among them, PET/CT plays a vital role because of its dual functional characteristics in metabolism and anatomy. Most studies believe that the application of PET/CT in the staging, treatment, and prognosis of different histological types of gastric lymphoma is feasible, such as gastric mucosa associated lymphoid tissue and diffuse large B cell lymphoma [27]. To distinguish gastric cancer and primary gastric lymphoma, it is undeniable that gastroscopy is the best method [28]. However, PET/CT shows its advantages for patients whose physical condition cannot tolerate examination or when the depth of lesions is large. Non-Hodgkin's lymphoma is the most common primary gastric lymphoma, among them, the proportion of high-grade lymphoma is higher than that of low-grade lymphoma. The pathological type of the former is mainly diffuse large B-cell lymphoma, and the latter is gastric. Mucosa-associated lymphoid tissue lymphoma is predominant [29]. Primary gastric lymphoma mainly infiltrates in the mucosa. Although its ulcer area is generally shallow, its growth range is wide, and its primary focus is large. Moreover, most lymphoma subtypes have a strong affinity for 18F-FDG, so the SUVmax of primary gastric lymphoma is higher. This fully demonstrates that SUVmax in PET/CT examination results provides an important diagnostic value for distinguishing gastric cancer and primary gastric lymphoma. In summary, PET/CT can identify stomach diseases in a more comprehensive way, especially in distinguishing gastric adenocarcinoma from primary gastric lymphoma, and can provide reliable semi-quantitative basis, so it has a wider application and higher clinical value.

Among the 110 patients in this retrospective study, 36 patients underwent radical surgery for gastric cancer in our hospital. More than $90 \%$ of the remaining patients were treated with radiation or by chemotherapy because they had reached advanced stages when the gastric malignant tumour was discovered, and a small number of the remaining patients chose to seek medical treatment in areas with better medical conditions. This also reflects the cause of the high mortality rate of gastric cancer in China, which lies in the failure of early detection, early diagnosis, and early treatment. In addition, the research hospital is located in north-western China. There is not much medical publicity, the local lack of awareness of medical treatment of the inhabitants, and financial or material resources are limited, which are the reasons why the tumour reaches advanced stages by the time of first visit to the hospital. The number of patients who have an opportunity of surgery or timely and proper treatment could have been higher if they were located in a developed area. All 36 patients received radical surgery, and postoperative pathology showed that they were all gastric adenocarcinoma. According to the histological types by the World Health Organisation, the patients were divided into high, medium, and low differentiation groups. After statistical analysis, the difference between these three groups of SUVmax was not statistically significant. Theoretically speaking, gastric cancer should show different uptake rates of 18F-FDG due to 
different degrees of differentiation, thus obtaining different SUVmax. However, because of the pathological reasons, this study could not subdivide histological types, such as intestinal type, diffuse type, and mixed type in Lauren's classification, hence, we could not get theoretical results. In general, the results of this study are consistent with those of Liu Y et al. [30], who believe that the degree of invasion of the gastric lining by the primary gastric tumour is related to the uptake of 18F-FDG, which shows different SUVmax. Therefore, PET/CT plays a high clinical diagnostic role in evaluating the T staging and progression of gastric cancer. Yoon J K [31] and others found that the depth of tumour invasion was an independent predictive factor of the high uptake rate of 18F-FDG by PET/CT in 229 patients with gastric cancer, so, the affinity of $18 \mathrm{~F}-\mathrm{FDG}$ in advanced gastric cancer is much higher than that in early gastric cancer. In this study, because T stage is an index used to describe the infiltration depth of malignant tumours, different infiltration depths mean different uptake rates of 18F-FDG. Furthermore, with the increase of infiltration depth, the uptake rate of 18F-FDG in the gastric mucosa, muscle layer, and serosa layer is higher, and the semi-quantitative SUVmax is larger. Similarly, in the postoperative pathological TNM staging, a later stage indicates a higher degree of local tumour infiltration, lymph node metastasis and distant metastasis. Correspondingly, the higher the SUVmax value in the PET/CT examination results. In addition, this study failed to find a statistically significant difference between $\mathrm{N}$ staging and SUVmax. As is well-known, lymphatic metastasis is the most important method of gastric cancer metastasis, $\mathrm{N}$ staging is a stage describing the number of lymph node metastases in gastric cancer. In the early stage, lymph node metastasis is mostly manifested as the metastasis of lymph nodes around the stomach, such as the lymph nodes of the cardia and lesser curvature of the stomach. As for the later stage of the disease, it is manifested as metastasis to distant lymph nodes or jumping metastasis, such as the left supraclavicular lymph node. The SUVmax does not show different results in different N stages, which may be due to the relatively high uptake rate of $18 \mathrm{~F}-\mathrm{FDG}$ in the primary focus of gastric cancer, which masks the uptake rate of surrounding metastatic lymph nodes [32]. Moreover, generally speaking, the volume of tumour focus must be larger than the volume of peri-gastric lymph nodes, so there is a certain influence on the uptake of lymph nodes. Therefore, the diagnostic value of PET/CT for lymph node metastasis may lie in the uptake rate of 18F-FDG in the local lymph node, rather than in the uptake rate of the primary tumour. The above results of the present study fully illustrate that PET/CT can provide an effective diagnostic value in the pathological staging of gastric cancer, which can better guide clinical treatment.

In terms of prognosis, relevant research findings at home and abroad are summarized as follows: Kim [33] et al. found that the difference between SUVmax and PFS was statistically significant through the study of 97 patients with gastric cancer examined by PET/CT prior to treatment, suggesting that SUVmax can be used as a good independent predictor of PFS, thus evaluating the prognosis of gastric cancer. Similarly, Park [34] et al. retrospectively analysed 82 cases of advanced gastric cancer and concluded that the high SUVmax group often suggests lower overall survival time (Overall Survival [OS]) and PFS, indicating that clinically, SUVmax can effectively predict the prognosis of patients with advanced gastric cancer. In addition, Grabinska [35] et al. and $\mathrm{Na}$ [36] et al. also studied and analysed the prognosis of patients with untreated gastric cancer, and found that many semi-quantitative parameters of PET/CT, 
including SUVmax, are closely related to OS, which has a clinical application value in evaluating prognosis. In the present study, due to objective factors such as short follow-up time and small sample size, the relationship between tumour differentiation and prognosis could not be found, but the relationship between SUVmax and survival time can still be found from this small sample study. This can be explained by the fact that from the medical professional point of view, the higher the uptake of $18 \mathrm{~F}$ FDG by the tumour focus, the higher the malignant degree and invasive ability of tumour, thus having a worse prognosis [37]. The small sample size of the present study may be the main reason for the bias of research results in factors such as tumour differentiation degree. Admittedly, the sample size and types of lesions in this study are relatively small, but there is some persuasiveness in the application value of $\mathrm{PET} / \mathrm{CT}$ in the diagnosis and prognosis of gastric cancer, and to some extent, a new research direction of $\mathrm{PET} / \mathrm{CT}$ in malignant tumours is defined.

In conclusion, for patients newly diagnosed with gastric cancer, PET/CT examination prior to treatment has obvious advantages in diagnostic and prognostic evaluations. PET/CT does not only provide reliable semi-quantitative basis for differentiating benign from malignant, but also has a great value for staging diagnosis and even pathological diagnosis. In terms of prognosis, the level of SUVmax also has a certain reference value in the survival time of patients after surgery, and becomes an independent risk factor for predicting prognosis, which can help doctors make better treatment plans in clinical aspects and achieve the purpose of improving prognosis. Admittedly, compared with other examinations, PET/CT examination is expensive and requires higher technical conditions at present. However, in the future, it will be more widely used in clinical practice, giving full play to its value in the diagnosis and prognosis of diseases.

\section{Abbreviations}

SUV

value of standard uptake value

$\mathrm{PET} / \mathrm{CT}$

positron emission tomography/computed tomography

18F-FDG

18F-labelled fluoro-2-deoxyglucose

CT

computed tomography

HR

hazard ration

$\mathrm{Cl}$

confidence interval

PFS

progression free survival

\section{Declarations}


Ethics approval and consent to participateПA retrospective analysis was carried out on the patients who were newly diagnosed with gastric cancer and underwent PET/CT examination between May 8, 2012 and July 14, 2020 in the Ningxia Medical University General Hospital. All medical records in this study have been informed and agreed by the patients. The authors have consented for publication.

Consent for Publication! Not applicable.

Availability of data and material[All the datasets generated or analyzed during the present study are included in this published article.

Competing interests:The authors declare no conflicts of interest.

Funding: This study was supported by projects from the Study on the Mechanism of Novel STAT3 Small Molecule Compounds Enhancing the Chemosensitivity of Triple Negative Breast Cancer with Positive EGFR Expression [grant numbers 2020AAC03178], the Study on the Mechanism of Novel HDAC6 Lnhibitors Against Colorectal Cancer Through HDAC6/STAT3 Signal Pathway [grant numbers 2020AAC03403] and Study on the Mechanism of HDAC Inhibitors Againstetastasis and Recurrence of Triple Negative Breast Cancer by STAT3/AKT/EMT Signaling Axis [grant numbers 816660500].

Authors' contributions: Study concept and design: Kairui Wang, Baozhen Wang, Qian Zhao, Jing Chen, and Tao Li; Acquisition of data: Kairui Wang, Baozhen Wang , Li Guo, Yongfeng Liang, Duowen Zhao; Analysis and interpretation of data: Kairui Wang and Baozhen Wang; Study supervision: Jing Chen and Tao Li.

Acknowledgements: Not applicable.

\section{References}

1. Wang FH, Shen L, Li J, et al. The Chinese Society of Clinical Oncology (CSCO): clinical guidelines for the diagnosis and treatment of gastric cancer[J]. Cancer Commun. 2019;39(1):10-1.

2. Wang W, Sun Z, Deng JY, et al. A novel nomogram individually predicting disease-specific survival after D2 gastrectomy for advanced gastric cancer[J]. Cancer Commun. 2018;38(1):23-4.

3. Chen W, Sun K, Zheng R, et al. Cancer incidence and mortality in China, 2014[J]. Chin J Cancer Res. 2018;30(1):1-12.

4. Katanoda K, Hori M, Matsuda T, et al. An updated report on the trends in cancer incidence and mortality in Japan, 1958-2013[J]. Jpn J Clin Oncol. 2015;45(4):390-401.

5. Siegel RL, Miller KD, Jemal A. Cancer statistics, 2016[J]. CA Cancer J Clin. 2016;66(1):7-30.

6. Chang F. Ji Rongzu. Clinical significance of combined detection of serum adiponectin, CEA, CA19-9 and CA72-4 in early screening of gastric cancer [J]. Journal of Modern Oncology. 2019;27(13):23226. 
7. Jung KW, Won YJ, Oh CM, et al. Cancer statistics in Korea: incidence, mortality, survival, and prevalence in 2014[J]. Cancer Res Treat. 2017;49(2):292-305.

8. Laterza MM, Pompella L, Petrillo A, et al. Efficacy of a triplet and doublet-based chemotherapy as first-line therapy in patients with HER2-negative metastatic gastric cancer: a retrospective analysis from the clinical practice[J]. Med Oncol. 2017;34(11):186-91.

9. Wang Shu L, Xuena D, Yao, et al. Use of 18F-FDG PET and PET/CT in differential diagnosis between recurrent or metastatic and chronic inflammation of esophageal cancer after treatment:A Meta analysis [J]. Journal of Modern Oncology. 2019;27(15):2740-4.

10. Almuhaimid TM, Lim WS, Roh JL, et al. Pre-treatment metabolic tumor volume predicts tumor metastasis and progression in high-grade salivary gland carcinoma[J]. J Cancer Res Clin Oncol. 2018;144(12):2485-93.

11. Gao Zhong-shan, Jia Zhi-bin, Zeng Xiang-gao, et al. 18F-FDG PETCT Maximum Correlation between SUV and PSA in Prostate Cancer Diagnosis and Treatment [J]. Chinese Journal of CT and MRI, 2018,16(8):122-124.

12. Jae $\mathrm{CH}$, Chan $\mathrm{K}$, Arthur $\mathrm{C}$, et al. The clinical implications of FDG-PET/CT differ according to histology in advanced gastric cancer[J]. Gastric Cancer,„2018,22(1):113-122.

13. Wang FH, Shen L, Li J, et al. The Chinese Society of Clinical Oncology (CSCO): clinical guidelines for the diagnosis and treatment of gastric cancer[J]. Cancer Commun,2019, 39(1): 4-6.

14. Camp RL, X-Tile. A New Bio-Informatics Tool for Biomarker Assessment and Outcome-Based CutPoint Optimization[J]. Clin Cancer Res,2004,10(21):7252-7259.

15. Wei ZUO. WANG Quan-shi. 18F-FDG PET/CT imaging in the diagnosis of gastric cancer and its effect on clinical decision [J]. The Journal of Practical Medicine,2015,31(1):39-42.

16. Jie CAO, Qi-chang YANG, Hong-bin LIU, et al. Expression of XAF1 and XIAP in gastric carcinoma tissues [J]. Chinese Journal of Cancer Prevention and Treatment,2019,26(07):468-472.

17. Li GOU, Feng GAO. Explore the Tumor staging and the diagnostic value of gastric cancer with $18 \mathrm{~F}-$ DFG PET/CT [J]. Xinjiang Medical Journal,2017,47(09):962-965.

18. Wu CX, Zhu ZH. Diagnosis and evaluation of gastric cancer by positron emission tomography [J]. World J Gastroenterol,2014,20(16): 4574-4585.

19. Fairweather M, Jajoo K, Sainani N, et al. Accuracy of EUS and CT imaging in preoperative gastric cancer staging[J]. J Surg Oncol. 2015;111(8):1016-20.

20. Qun ZHAO, Yong LI, Liang WANG, et al. Application of MSCT in evaluating the effect of neoadjuvant chemotherapy in advanced gastric cancer [J]. Chinese Journal of Cancer Prevention Treatment. 2019;26(01):43-6.

21. Jin ZHOU, Dongya ZHOU, Yin ZHANG, et al. Correlation of 18F-FDG PET/CT metabolic parameters and clinicopathological factors of esophageal cancer [J]. Chinese Journal of Medical Imaging Technology,2018,34(07):1024-1027. 
22. Brenkman HJF, Gertsen EC, Vegt E, et al. Evaluation of PET and laparoscopy in Staging advanced gastric cancer: A multicenter prospective study (PLASTIC-study)[J]. BMC Cancer. 2018;18(1):1-7.

23. Choi BH, Song HS, An YS, et al. Relation Between Fluorodeoxyglucose Uptake and Glucose Transporter-1 Expression in Gastric Signet Ring Cell Carcinoma[J]. Nuclear Medicine \& Molecular Imaging, 2011, 45(1):30-35.

24. Lim JY, Yoon SO, Seol SY, et al. Overexpression of the M2 isoform of pyruvate kinase is an adverse prognostic factor for signet ring cell gastric cancer[J]. World J Gastroentero. 2012;18(30):4037-43.

25. Gaofeng S, Guixia P, Ye P, et al. Diagnosis and differentiation with 18F-FDG PET-CT: gastric lymphoma and gastric carcinoma [J]. Journal of Medical Imaging. 2013;23(2):247-51.

26. Li XF, Fu Q, Dong YW, et al. 18F-fluorodeoxyglucose positron emission tomography/computed tomography comparison of gastric lymphoma and gastric carcinoma[J]. World J Gastroentero. 2016;22(34):7787-96.

27. Yi JH, Kim SJ, Choi JY, et al. 18F-FDG uptake and its clinical relevance in primary gastric lymphoma[J]. Hematol Oncol. 2010;28(2):57-61.

28. Wu J, Zhu H, Li K, et al. 18F-fluorodeoxyglucose positron emission tomography/computed tomography findings of gastric lymphoma: Comparisons with gastric cancer[J]. Oncol Lett. 2014;8(4):1757-64.

29. Zhang YA, Wei Zheng, Li J,et al.Molecular pathogenesis of lymphomas of mucosa-associated lymphoid tissue-from(auto)antigen driven selection to the activation of NF- $\otimes \mathrm{B}$ signaling[J].Science China(Life Sciences),201558(12):1246-1255.

30. Liu Y, Zheng D, Liu JJ, et al. Comparing PET/MRI with PET/CT for Pretreatment Staging of Gastric Cancer[J]. Gastroent Res Pract. 2019;2019:1-11.

31. Yoon JK, Byun C, Jo KS, et al. Clinicopathologic parameters associated with the FDG-avidity in staging of early gastric cancer using 18F-FDG PET[J]. Med (Baltimore). 2019;98(31):16690-7.

32. Ying CUI, Jian ZHANG, Chang-jin ZUO. The evaluation of PET/CT in diagnosis of gastric cancer and treatment [J]. Journal of Medical Imaging,2014,24(09):1631-1635.

33. Kim J, Lim ST, Na CJ, et al. Pretreatment F-18 FDG PET/CT Parameters to Evaluate Progression-Free Survival in Gastric Cancer[J]. Nucl Med Mol Imaging. 2014;48(1):33-40.

34. Park JC, Lee JH, Cheoi K, et al. Predictive value of pretreatment metabolic activity measured by fluorodeoxyglucose positron emission tomography in patients with metastatic advanced gastric cancer: the maximal SUV of the stomach is a prognostic factor[J]. Eur J Nucl Med Mol Imaging. 2012;39(7):1107-16.

35. Grabinska K, Pelak M, Wydmanski J, et al. Prognostic value and clinical correlations of 18fluorodeoxyglucose metabolism quantifiers in gastriccancer[J]. World J Gastroent. 2015;21(19):5901-9.

36. NA SJ, Joo HO, Park JM, et al. Prognostic value of metabolic parameters on preoperative 18FFluorodeoxyglucose positron emission tomography/ computed tomography in patients with stage III gastric cancer[J]. Oncotarget. 2016;7(39):63968-80. 
37. Song JL, Wang XJ, Yang Z. Application of ${ }^{18} \mathrm{~F}$-fluorodeoxyglucose PET/CT in prediction of prognosis of advanced gastric cancer[J]. Oncoradiology,2018, 27(2):60-64.

Figures

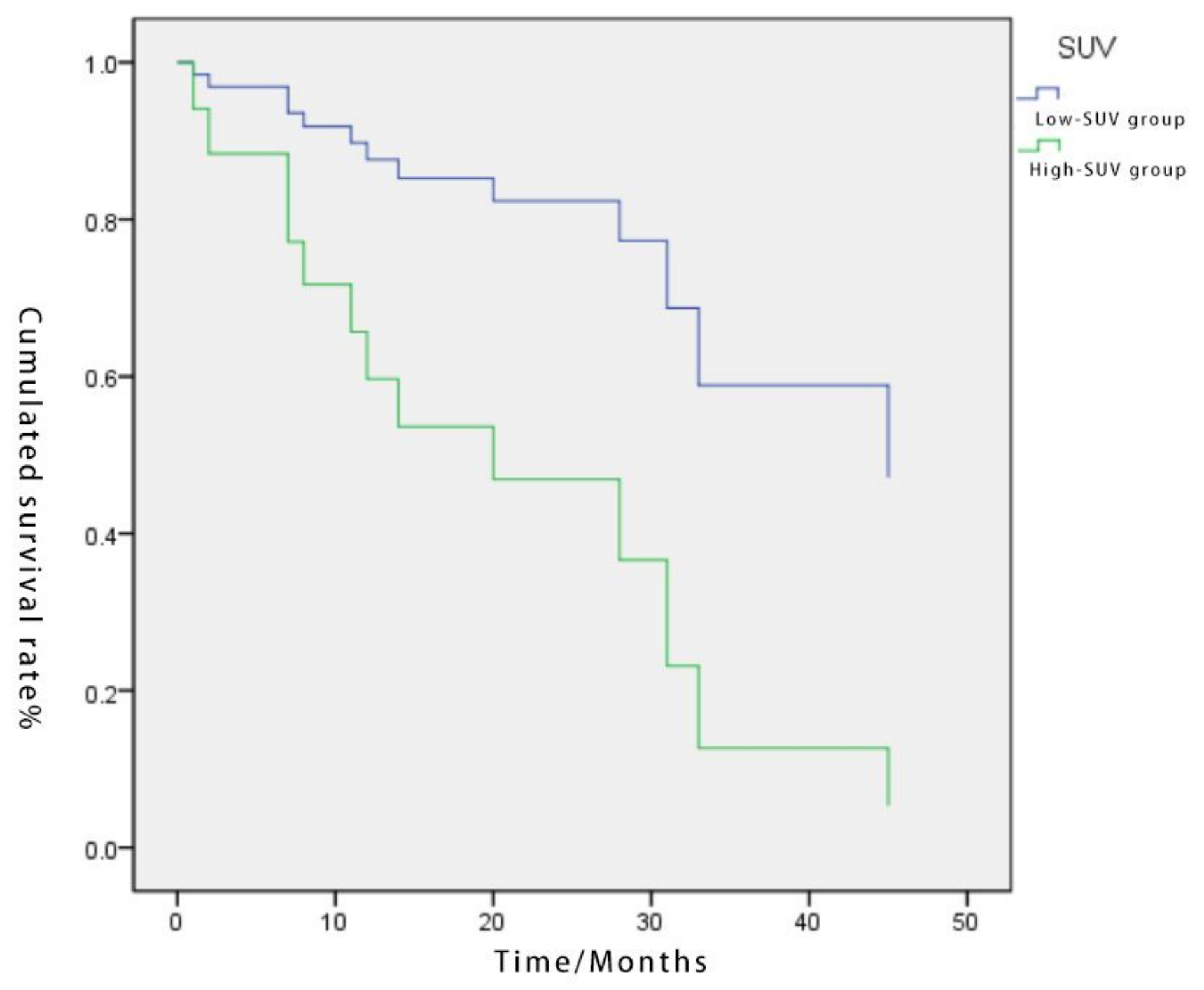

Figure 1

Kaplan-Meier survival analysis chart of different SUVmax values 


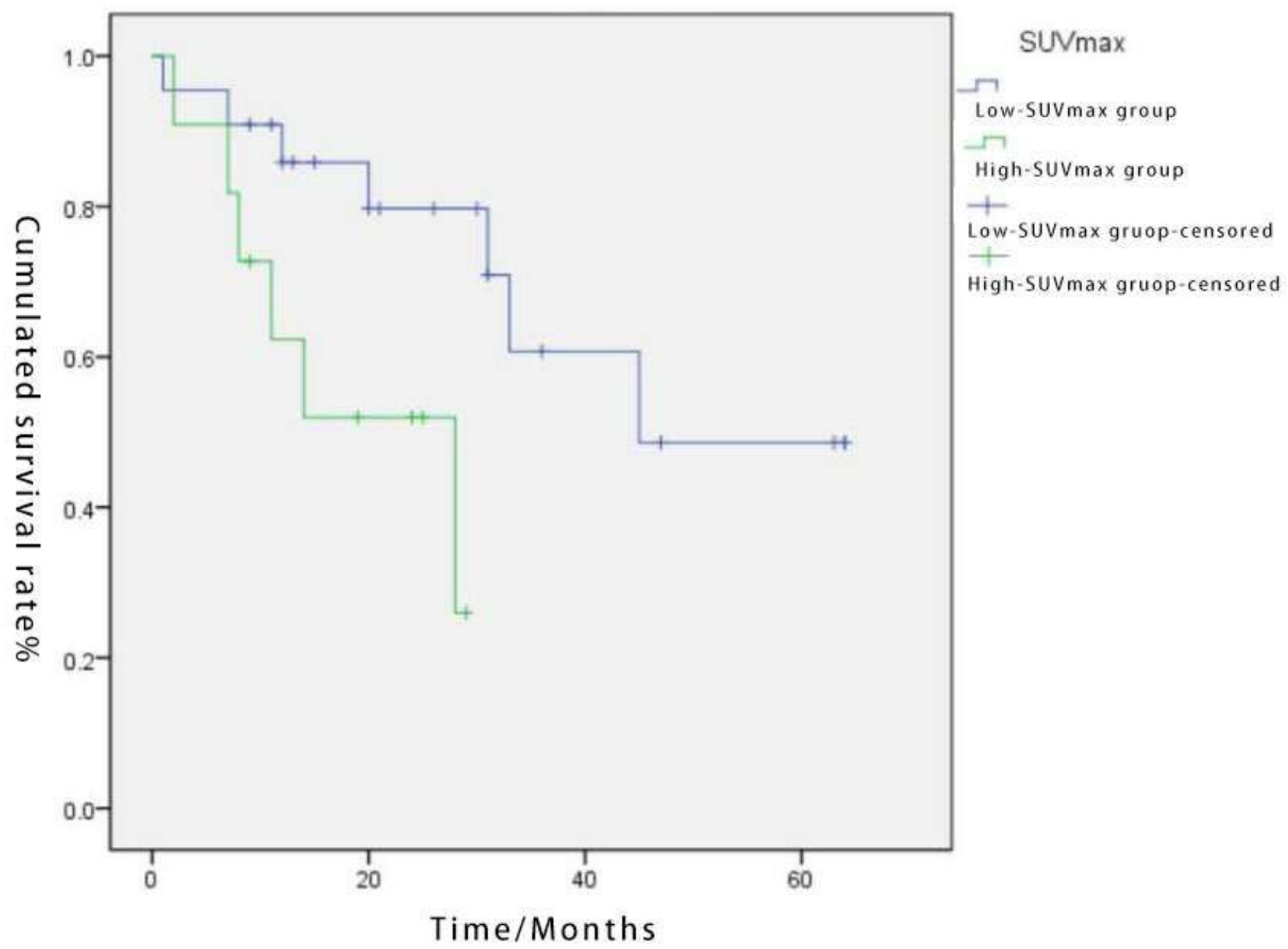

Figure 2

COX survival analysis chart 